\title{
NEGATIVELY AND POSITIVELY POLARIZED HYDROGEN ADSPECIES ON THIN PLATINUM FILMS
}

\author{
E. Nowicka, Z. Wolfram and R. Duś \\ Institute of Physical Chemistry, Polish Academy of Sciences \\ Kasprzaka 44/52, 01-224 Warszawa, Poland \\ (Received June 3, 1991; in final form September 4, 1991)
}

\begin{abstract}
Hydrogen adsorption on thin platinum films under isothermal conditions within the temperature and pressure intervals $78-298 \mathrm{~K}$ and $10^{-10}-10^{-2}$ Torr has been studied measuring simultaneously surface potential $(S P)$ and pressure $(P)$, by means of a sensitive, short response time apparatus. Two forms of hydrogen deposit of different electrical character have been distinguished. The first one arising at the beginning of adsorption decreases the surface potential, the second one following it increases the $S P$ due to positively polarized adspecies formation. This positively polarized form is inhomogeneous as concerns binding energy. Hydrogen uptake associated with the decrease of $S P$ becomes larger with increase of temperature. It seems that this $\beta^{-}$form of the adsorbate stabilizes the positively polarized adspecies on the surface.
\end{abstract}

PACS numbers: 82.65-i, 73.60.Aq

\section{Introduction}

In contrast to many transition metals, platinum interaction with hydrogen does not lead to platinum hydride formation. Solubility of hydrogen in the bulk of platinum is low [1], however the platinum surface is active for dissociative hydrogen adsorption. IIydrogen adsorbate on platinum surfaces is complex and consists of several different states [2-9]. Adspecies corresponding to these states are placed on different adsorption sites [2-4], they differ in binding energy [4-8], and in electrical character [4, 7-9]. Strongly adsorbed adatoms and weakly bound molecular hydrogen adspecies have been observed on thin platinum film surfaces at a pressure exceeding $10^{-4}$ Torr $[8,10,11]$ while only atomic hydrogen has been found on the surfaces of low index single crystal planes at pressures of the order of $10^{-8}$ Torr $[6,7]$. On a flat $\mathrm{Pt}$ (111) surface the positively polarized hydrogen adspecies $\beta^{+}$are formed [6] (the positive pole of the dipole placed out of the surface) increasing the surface potential (SP). IIowever on stepped [7] and on reconstructed surfaces of single crystals [4] and also on thin platinum films $[8,9]$ a decrease of 
surface potential arises at the beginning of adsorption and followed by an increase of $S P$ when the coverage increases. The decrease of surface potential can be observed for two reasons: (1) negatively polarized hydrogen adspecies formation due to reversal charge transfer, which is possible at surface steps, vacancies and kinks [12], or (2) positively polarized adspecies placing below the surface image plane [4, 13-15]. On the surface of thin platinum films both effects can be expected. The question arises how far the presence of negatively polarized hydrogen adspecies, or of positively polarized adspecies placed below the surface image plane, influences the properties of the adatoms present at the platinum surface. We announce our first experimental results concerning this question.

\section{Experimental}

The studies were carried out by measuring simultaneously surface potential changes $(\triangle S P)$ and pressure $(P)$ during hydrogen adsorption performed at constant temperature, and in the course of isothermal desorption caused by evacuation of the system. An UIIV glass system capable of reaching routinely $1-2 \times 10^{-10}$ Torr was used. Thin platinum films were deposited under this pressure on the wall of the cylindrical static capacitor [6] maintained at $78 \mathrm{~K}$, by evaporation of fine platinum wire (Johnson-Matthey, grade 1) wound around a tungsten heater. Thin films were sintered after the deposition at $330 \mathrm{~K}$ for $20 \mathrm{~min}$ and then a temperature required for the adsorption experiment was maintained. Some of the films were tested by means of spectral analysis. The films were free of tungsten traces. The geometrical area of the films was $135 \mathrm{~cm}^{2}$ and their thickness $\sim 10^{-7} \mathrm{~m}$. The roughness factor determined in separate experiments by means of hydrogen-oxygen titration [17] was $16 \pm 2$. Spectroscopically pure hydrogen, purified additionally by diffusion through a palladium thimble was used. In order to avoid atomisation of hydrogen on the hot filament and the pumping effect of the ionization gauge which influences volumetric calculations, in the course of hydrogen adsorption, the pressure was measured using an ultrasensitive, short response time (1 decade/s) Pirani-type gauge, capable of working within the range $1 \times 10^{-6}-1 \times 10^{-1}$ Torr. Surface potential measurements were performed using the previously described static capacitor gauge with an electronic circuit of short overall response time $(0.1 \mathrm{~s})$ and low noise level $(0.2 \mathrm{mV})$ [16]. That made it possible to study surface potential changes during fast adsorption-desorption processes.

Two types of experiments were performed: (i) static experiments with an introduction of hydrogen in successive calibrated doses into the static capacitor disconnected from pumps, (ii) dynamic experiments with a constant flow of hydrogen through a calibrated capillary into the static capacitor disconnected from pumps.

From the static experiments the following relation are obtained:

1) The thermodynamic isotherm $P_{\text {eq }}=f_{1}\left(n_{a}\right)_{T}$, where $P_{\text {eq }}$ is equilibrium

pressure corresponding to hydrogen uptake $n_{a}$.

2) The surface potential isotherm $\Delta S P=f_{2}\left(n_{a}\right)_{T}$.

On the basis of the dynamic experiments one obtains the following relations: $P(t)=f_{3}(t)_{T}$, and $\Delta S P=f_{4}(t)_{T}$, where $P(t)$ is hydrogen pressure at time $t$ of 
flow. On the basis of the static experiments it is possible to differentiate between the distinguished states of the adsorbate, while the data collected in the dynamic experiments allow to calculate the sticking probability factor $S(\theta)$ for these states. It is easy to notice that combining the functions $f_{1}, f_{2}, f_{3}$ and $f_{4}$ the effective adsorption rate can be determined

$$
\frac{\mathrm{d} n_{a}}{\mathrm{~d} t}=S(\theta) A Z\left[P(t)-P_{\text {eq }}\right] \text {, }
$$

where $A$ is the true area of the film, and $Z$ is the collision factor. This method offers the possibility to determine $S(\theta)$ for weakly adsorbed states arising at comparatively high pressure, e.g. of the order of $10^{-2}$ Torr, since the change of population is monitored directly on the surface by measuring $\triangle S P(t)$ instead of being deduced from the change in gas phase density as in the classic Wagener flow method. The construction of the static capacitor [16] enables one to carry out the sticking probability measurements also by means of the Wagener method. In this case $S(\theta)$ is given by the equation

$$
S(\theta)=\frac{L-(V / k T) \mathrm{d} P / \mathrm{d} t}{L+Z A\left[P(t)-P_{\mathrm{eq}}\right]},
$$

where $L$ is the flux of hydrogen and $V$ is the volume of the capacitor. Measurements of this type were performed at pressures of the order of $10^{-6}$ Torr, to compare them with the static capacitor method. The agreement was good. Another advantage of the static capacitor was the possibility to study isothermal desorption of weakly adsorbed adspecies caused by evacuation. $\triangle S P$ in the course of the desorption is recorded as a function of time $\Delta S P=f_{5}(t)$. Combining $f_{2}$ and $f_{5}$ one can get the equation of the rate of decrease of population in the course of the isothermal desorption $-\mathrm{d} n_{a} / \mathrm{d} t=f_{6}(t)$, and next to apply the well-known kinetic relations to examine the process.

\section{Results and discussion}

Surface potential changes measured as the result of the introduction of successive doses of hydrogen into the static capacitor with thin platinum films maintained at $78 \mathrm{~K}$ and $298 \mathrm{~K}$ during the static experiments are shown in Fig. 1. The coverage was calculated assuming that at monolayer the adsorption stoichiometry is $1: 1$ (one atom of platinum on the surface per one adatom) [4], and the average density of platinum surface atoms is $1.25 \times 10^{15} \mathrm{~cm}^{-2}$ [18]. The geometrical area and the roughness factor of thin films were known $\left(135 \mathrm{~cm}^{2}\right.$ and $16 \pm 2$ respectively). In Fig. $1 \triangle S P$ is also to be seen due to isothermal desorption caused by evacuation. Surface potential isotherms are presented in Fig. 2. It can be seen in Figs. 1 and 2 that the main features of $\triangle S P$ dependence on coverage are similar at all applied temperatures, but that the details differ significantly. At the beginning of adsorption several doses of hydrogen introduced into the static capacitor decreased the surface potential. Further doses increased $S P$ due to positively polarized adspecies formation. Hydrogen uptake corresponding to the decrease of $S P$ is larger at higher temperatures (Fig. 2). This suggests activated adsorption in this step of the process. We have observed that strong sintering of thin platinum 


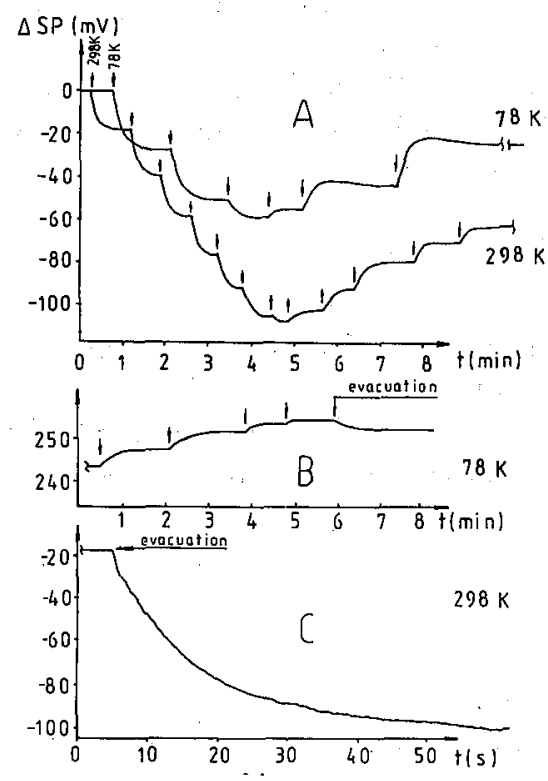

Fig. 1. Surface potential changes $\triangle S P$ caused by hydrogen adsorption on thin platinum films at $78 \mathrm{~K}$ and $298 \mathrm{~K}$ in the course of the static experiments. Arrows indicate introduction of the successive liydrogen doses. The graph a shows $\Delta S P$ at the beginning of adsorption, while graplis b and c $\triangle S P$ at the end of adsorption and during the isothermal desorption caused by evacuation of the system.

films reduced significantly, but not completly the uptake of hydrogen adsorbed with a decrease of $S P$. We suppose that this effect corresponds to the diminishing of concentration of surface imperfections, while penetration of positively polarized adspecies below the surface image plane still occurs. This process is probably activated [4]. Indeed, the surface potential increases as the result of several successive doses leading to positively polarized adspecies is monotonic at $298 \mathrm{~K}$, but at $78 \mathrm{~K}$ positive $S P$ transients can be seen (Fig. 1). These transients are even more pronounced at $195 \mathrm{~K}$. We suppose that the positive $S P$ transients are associated with incorporation of positively polarized adspecies below the surface image plane. The rate of this process is higher at $195 \mathrm{~K}$ than at $78 \mathrm{~K}$, and that allows to estimate the activation energy for the incorporation. The incorporation can be considered as a relaxation process [19] and should be described by the first order kinetic equation. We have examined that all $S P$ transients are well-described by this type of equation. Assuming that within one hydrogen dose there is a proportionality between $\triangle S P$ and $n_{a}$, we estimated the activation energy for incorporation of the positively polarized hydrogen adspecies below the platinum surface image plane as equal to $1.2 \mathrm{~kJ} / \mathrm{mole}_{2}$. This low activation energy barrier can be easily jumped over at $298 \mathrm{~K}$ and the subsurface position is reached quickly, thus $S P$ transients are not recorded. It should be emphasised that the amount of hydrogen 
incorporated below the platinum surface image plane is small, smaller than the monolayer, in contrast with large amount of hydrogen absorbed in the volume of transition metals forming hydrides [19]. Within the temperature interval 78-298 K hydrogen uptake corresponding to $\theta=1$ can be reached (Fig. 2) when an appropriate pressure is applied. It can be seen in Fig. 1 that evacuation of the static

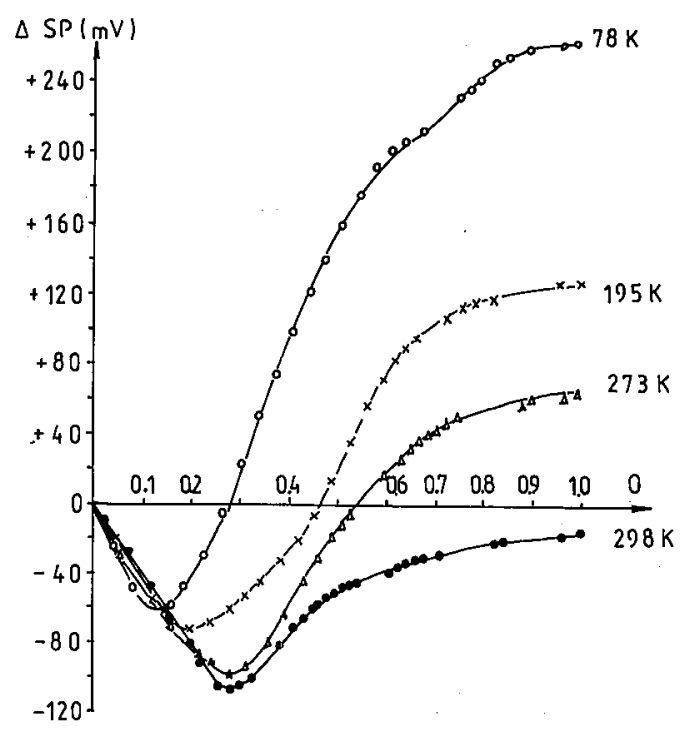

Fig. 2. Surface potential isotherms for liydrogen adsorption on thin platinum films at $78 \mathrm{~K}, 195 \mathrm{~K}$ and $298 \mathrm{~K}$.

capacitor at $78 \mathrm{~K}$ leads to desorption of a small part of the positively polarized adsorbate $(\sim 10 \%)$ (Fig. 1b), while at $298 \mathrm{~K}$ a large part of the deposit can be removed (Fig. 1c). On the basis of lifetime considerations we conclude that $\sim 10 \%$ of the adsorbate is bound with an energy lower than $20 \mathrm{~kJ} / \mathrm{mole} \mathrm{H}_{2}$. It can be seen in Fig. 2 that the composition of the hydrogen adsorbate that means the ratio of hydrogen uptake decreasing $S P$ to the uptake increasing $S P$, is similar at $273 \mathrm{~K}$ and $298 \mathrm{~K}$, but differs strongly from the one obtained at $195 \mathrm{~K}$ and $78 \mathrm{~K}$. Having experimentaly determined $P_{\mathrm{eq}}=f_{1}\left(n_{a}\right)$ functions at $273 \mathrm{~K}$ and $298 \mathrm{~K}$ we estimated the isosteric heat of adsorption $Q(\theta)$ and the differential entropy $\bar{S}(\theta)$, according to the well-known equations

$$
\begin{aligned}
& Q(\theta)=R\left[\frac{\mathrm{d} \ln P_{\mathrm{eq}}}{\mathrm{d}(1 / T)}\right]_{\theta}, \\
& \bar{S}(\theta)=S_{0}-Q(0) / T-R \ln \left(P_{\mathrm{eq}} / P_{0}\right),
\end{aligned}
$$

where $S_{0}$ is the standard molar entropy of hydrogen at pressure $P_{0}$. Results are shown in Fig. 3.

In this figure the dependence of $\ln P_{\mathrm{eq}}$ on $1 / T$ at constant $\theta$ is also presented. One should expect a straight line dependence of $\ln P_{\text {neq }}$ on $1 / T$ at constant $\theta$, if 


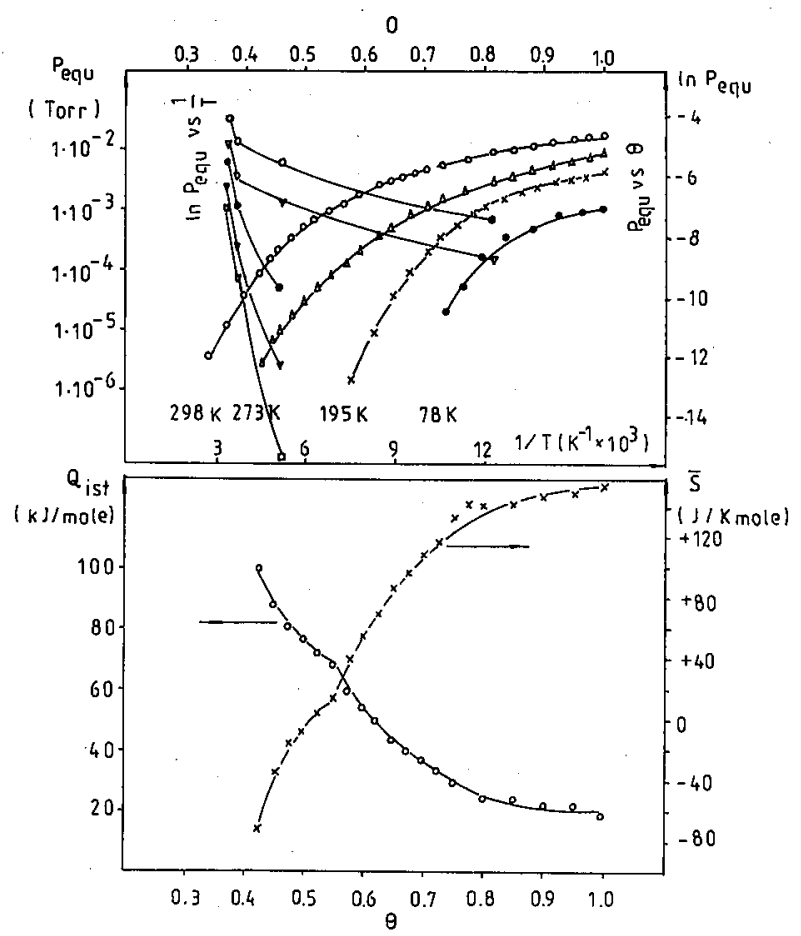

Fig. 3. $P_{\mathrm{eq}}=f_{1}(\theta)_{T}$ functions for hydrogen adsorption on thin platinum films at 78 $\mathrm{K}, 195 \mathrm{~K}, 273 \mathrm{~K}$ and $298 \mathrm{~K}$. The relations $\ln P_{\text {eq }}$ vs. $1 / T$ are inserted for $\theta=0.60,0.65$, $0.70,0.75$ and 0.80 (from left to right). In the lower part of the figure the dependences of isosteric heat of adsorption and difTrential entropy of adsorption on coverage for the $\beta^{+}$hydrogen adspecies are presented.

within the examined temperature interval, the same state of the adsorbate exists. It can be seen in Fig. 3 that this is not the case for positively polarized hydrogen adspecies on thin platinum film within temperature interval 78-298 K. The equilibrium pressure calculated for $0=0.6$ at $195 \mathrm{~K}$ on the basis of Eq. (3) taking $Q(\theta)$ estimated within $273-298 \mathrm{~K}$ is of two orders of magnitude lower than that measured experimentally. The difference is even larger at higher $\theta$. It seems that the preadsorbed hydrogen adspecies decreasing $S P$ can stabilize the $\beta^{+}$form of the deposit on thin platinum surface. Since this effect is very strong it can not be a result of lateral interaction between dipoles but rather arises due to the change of electron density around platinum surface atoms.

On the basis of the dynamic experiments it was possible to calculate the sticking probability coeflicient for weakly adsorbed $\beta^{+}$adspecies existing on thin platinum film surface at pressure interval $1 \times 10^{-6}-1 \times 10^{-2}$ Torr. These adspecies desorb during isothermal desorption at $273 \mathrm{~K}$, and partially even at $195 \mathrm{~K}$. At these experiments hydrogen was introduced at constant flow into the static capacitor with the platinum film precovered by strongly adsorbed species deposited 
in the course of the static experiments. Results of calculations are shown in Fig. 4. Some points calculated on the basis of Wagener method are inserted into the graph. It can be noticed that at constant $\theta$ the sticking probability coefficients increase

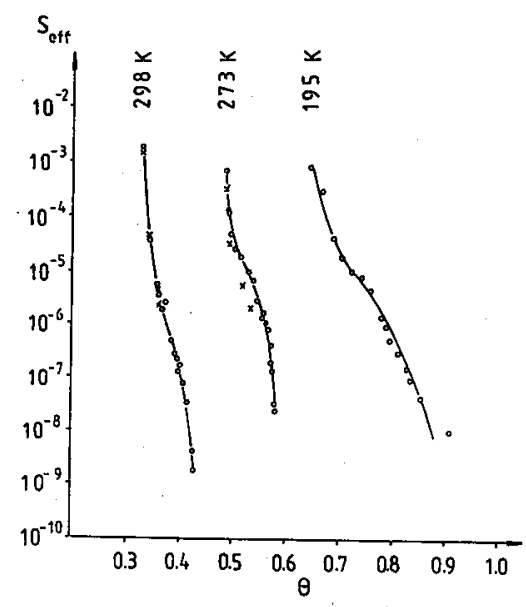

Fig. 4. Sticking probability coefficients for weakly adsorbed $\beta^{+}$adspecies on thin platinum films precovered with strongly bounded hydrogen adspecies. Circles correspond to the dynamic experiments described in this work, while crosses correspond to the results obtained by means of classic Wagener method.

with temperature decrease. That suggests an unactivated adsorption process retarded by increase of temperature dependent effective area occupied by mobile $\beta^{+}$ adatoms. Sticking probability for hydrogen adspecies strongly adsorbed on thin platinum films was previously measurede by Lisowski [20] using classic Wagener method. The extrapolated data coincide well with our results obtained at pressure of the order of $10^{-6}$ Torr. Examination of isothermal desorption of the $\beta^{+}$adspecies (Fig. 1) showed that simple kinetic equations did not fit the experimental data. This can be in fact expected since heat of adsorption changes strongly and nonlinearly with coverage (Fig. 3).

\section{Conclusions}

i) Hydrogen adsorption on thin platinum films within temperature interval 78-298 K leads to decrease of surface potential followed by its increase at higher coverage.

ii) The decrease of $S P$ is caused by formation of the negatively polarized adatoms $\beta^{-}$at surface imperfections and penetration of the positevely polarized adatoms $\beta^{+}$below the surface image plane. The last process is an activated one.

iii) Hydrogen deposit composition on thin platinum film surface (concentration of the $\beta^{-}$and the $\beta^{+}$adspecies) depends on adsorption temperature.

iv) The presence of the $\beta^{-}$adspecies stabilizes the $\beta^{+}$adatoms on thin platinum film surface. 


\section{Acknowledgements}

The authors have pleasure in acknowledging the master glass-work done by Mr. J. Biechoński and Mr. R. Bojarski which made it possible to carry out the studies.

\section{References}

[1] N.A. Gałakinowa, Vodorod v Metallakh, Metallurgia, Moskva 1967.

[2] P.R. Norton, J.A. Daviss, D.P. Jackson, N. Matsunami, Surf. Sci. 85, 269 (1979).

[3] P.R. Norton, D.K. Creber, J.A. Davies, J. Vac. Sci. Technol. 17, 149 (1980).

[4] B. Pennemann, K. Oster, K. Wandelt, submitted to Surf. Sci.

[5] R. Lewis, R. Gomer, Surf. Sci. 17, 333 (1969).

[6] K. Christmann, G. Ertl, T. Pignet, Surf. Sci. 54, 365 (1976).

[7] K. Christmann, G. Ertl, Surf. Sci. 60, 365 (1976).

[8] R. Duś, F.C. Tompkins, J. Chem. Soc. Faraday Trans. I 71, 930 (1975).

[9] J.C.P. Mignolet, J. Chem. Phys. 54, 19 (1957).

[10] R. Suhrmann, G. Wedler, II. Gentsch, Z. Phys. Chem. (Neue Folge) 17, 350 (1958).

[11] V. Ponec, J. Catalysis 6, 362 (1966).

[12] J. Anzelm, Surf. Sci. 108, 561 (1981).

[13] R. Engstrom, W. Tsai, W.M. Weinberg, J. Chem. Phys. 87, 3104 (1987).

[14] E. Kirsten, G. Parschau, W. Stocker, K.H. Rieder, Surf. Sci. 231, L183 (1990).

[15] R. Duś, E. Nowicka, Z. Wollram, Surf. Sci. 216, 1 (1989).

[16] E. Nowicka, R. Duś, Surf. Sci. 144, 665 (1984).

[17] R. Duś, W. Lisowski, Surf. Sci. 59, 141 (1976).

[18] D. Brennan, D.O. Hayward, B.M.W. Trapnell, Proc. R. Soc. Lond. A 256, 81 (1960).

[19] E. Nowicka, Z. Wolfram, R. Duś, Surf. Sci. 247, 248 (1991).

[20] W. Lisowski, Appl. Surf. Sci. 31, 451 (1988). 